\title{
Effects Of Ion Energy On Nitrogen Plasma Immersion Ion Implantation In UHMWPE Polymer Through A Metal Grid
}

Cite as: AIP Conference Proceedings 866, 241 (2006); https://doi.org/10.1063/1.2401504

Published Online: 01 December 2006

M. Ueda, R. M. Oliveira, J. O. Rossi, C. M. Lepienski, and W. A. Vilela
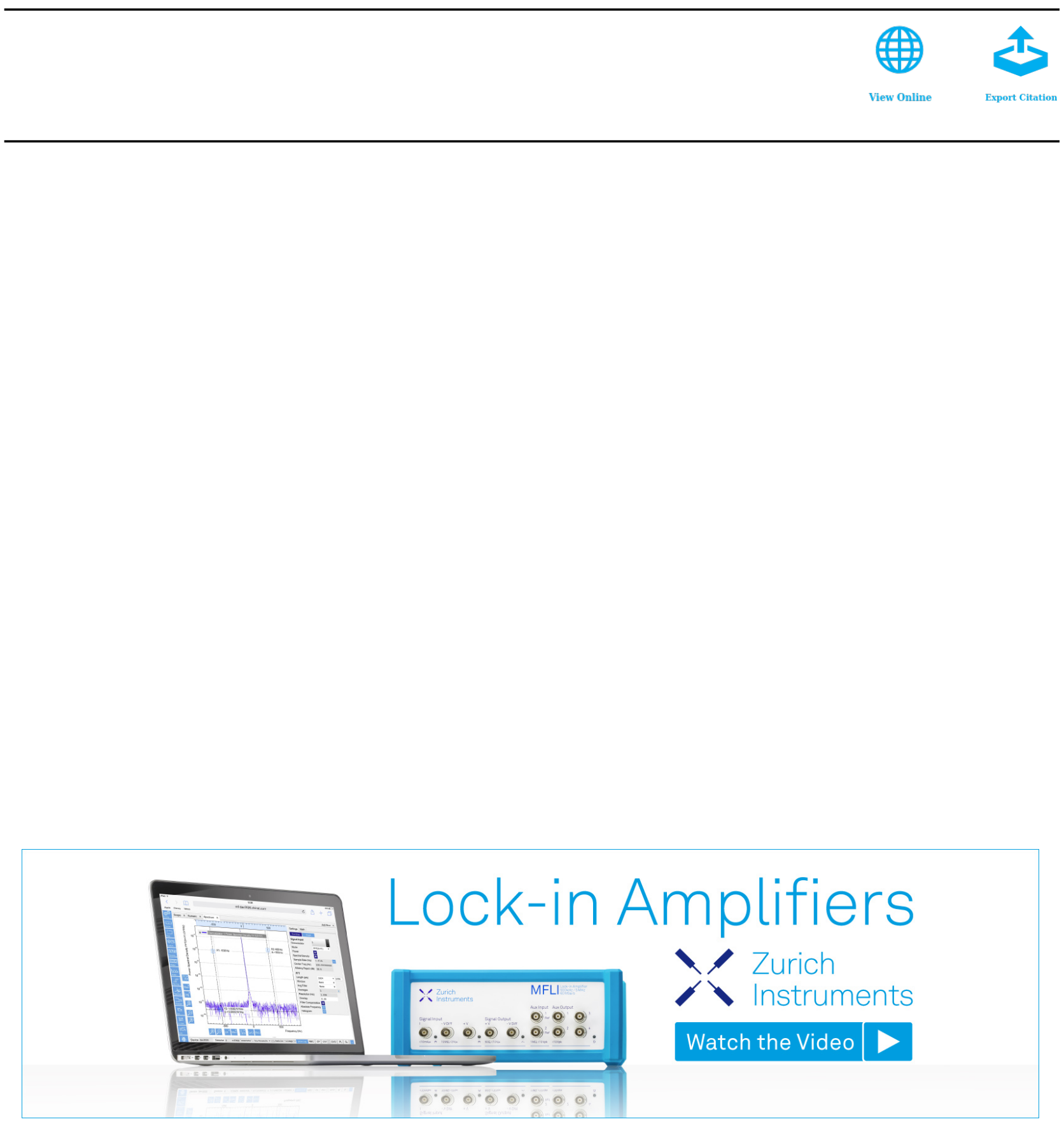


\title{
Effects Of Ion Energy On Nitrogen Plasma Immersion Ion Implantation In UHMWPE Polymer Through A Metal Grid
}

\author{
M. Ueda ${ }^{1}$, R.M. Oliveira ${ }^{1}$, J.O. Rossi ${ }^{1}$, C.M. Lepienski ${ }^{2}$, and W.A. Vilela ${ }^{3}$ \\ ${ }^{1}$ Associated Laboratory of Plasma, National Institute for Space Research, \\ Av. dos Astronautas 1758, São José dos Campos, São Paulo, Brazil \\ ${ }^{2}$ Department of Physics, Federal University of Paraná, Curitiba, Brazil \\ ${ }^{3}$ Associated Laboratory of Materials and Sensors, National Institute for Space Research, \\ São José dos Campos, SP, Brazil
}

\begin{abstract}
Herein, we consider the potential application of plasma immersion ion implantation (PIII) for treatment of polymer surfaces. This paper presents some experimental data for ultra-high molecular weight polyethylene (UHMWPE) implanted with nitrogen using PIII process. This polymer is widely used in medical prosthesis and PIII treatment has revealed to be an ease and cheap way to improve the lifetime of prosthesis made with UHMWPE. Here we show the latest results for UHMWPE surface treatment obtained with the use of a high voltage pulser of $100 \mathrm{kV} / 200 \mathrm{~A}$ based on coaxial Blumlein technology.
\end{abstract}

Keywords: plasma implantation, Blumlein line, high voltage, nitrided surfaces, ultra-high molecular weight polyethylene, and diamond-like carbon

PACS: $52.77 . \mathrm{Dq}$

\section{INTRODUCTION}

To explore new operating regimes in surface treatment by plasma immersion ion implantation (PIII), high voltage pulses of the order of $100 \mathrm{kV}$ are needed. For instance, high energy is essential to obtain great depth of penetration of ion species into the surface of polymers, which increases the hardness factor and wear resistance of polymeric components under certain manufacturing processes [1]. On the other hand, working with short pulses (around $1 \mu \mathrm{s}$ ) leads to the improvement of the conformal implantation of small pieces by decreasing the width of plasma sheath. For instance, using shorter pulse durations $(\approx 1 \mu \mathrm{s})$, higher currents $(>100 \mathrm{~A})$, and highdensity plasma can produce sheath thickness below $2.5 \mathrm{~cm}$ as described elsewhere [2].

Recent studies, conducted by the plasma group at INPE, has shown a tremendous potential of the PIII treatment [3],[4] on improvement of mechanical properties of a ultra-high molecular weight polyethylene (UHMWPE), a kind of polymer widely used in traditional and modern industries. As an example of the traditional case, we can cite the protection layer in mine ore transporting dump trucks, valves or connecting tubes in food processing or chemical factories, etc. The most representative modern application of UHMPWE is probably the acetabular cap used in repairing component of femoral prosthesis. Despite its excellent properties regarding biocompatibility, machineability, and mechanical qualities, the extremely demanding conditions of real prosthesis operations in the human body require further improvements in surface properties, especially with respect to wear. Nitrogen plasma implantation (PIII) applied to UHMWPE material synthesizes a DLC layer on its surface with improved mechanical properties [3]. To avoid severe charging dielectric effects during PIII treatments of such non-conducting materials, we used a metal grid during ion implantation process. We found that the ion energy affects the homogeneity of the treated layer, being favorable for higher energies (for the range of 5 to $15 \mathrm{keV}$ with pulse duration of the order of $10 \mu \mathrm{s}$ ). Much higher implantation energies up to $50 \mathrm{keV}$ with very short pulses of $1.2 \mu \mathrm{s}$ were tested also, and analyses based on Raman spectroscopy carried out for identifying diamond-like carbon (DLC) distribution over the surface.

In next section, we present two experimental setups used to carry out the tests while in section III we show the latest results obtained so far.

CP866, Ion Implantation Technology,

edited by K. J. Kirkby, R. Gwilliam, A. Smith, and D. Chivers

(C) 2006 American Institute of Physics 978-0-7354-0365-9/06/\$23.00 


\section{EXPERIMENTAL SET-UP}

For the implantation tests, we used two different experimental set-ups. For the first tests carried out with lower voltages and long pulse duration $(5 \mathrm{kV} / 15 \mathrm{kV} \& 10 \mu \mathrm{s})$, we used the system depicted in Fig. 1. In this case, a hard-tube (HT) pulser with maximum V/I capability of $30 \mathrm{kV} / 30 \mathrm{~A}$ (connected to the PIII system as shown in Fig. 1) was able to produce pulse duration in the range of $5-10 \mu \mathrm{s}$ at a repetition frequency of $100 \mathrm{~Hz}$ [5], [6]. A simple power supply capable of providing maximum V/I parameters of $900 \mathrm{~V} / 0.6 \mathrm{~A}$ gives output power for the DC glow discharge. The nitrogen plasma discharge initiated between the cylindrical stainless steel rod and the neck connected to the PIII cylindrical chamber with $27 \mathrm{~cm}$ diameter and $47 \mathrm{~cm}$ length as shown in Fig. 1. The stainless steel rod linked to a copper feed-through connector applied the negative high voltage pulse from the HT pulser. We typically run the discharges at powers of $180 \mathrm{~W}$, with corresponding discharge voltages of $600 \mathrm{~V}$ and currents of approximately of 0.3 A. Under this conditions the plasma potential near the center of the PIII plasma chamber reached $350 \mathrm{~V}$, which in some cases can be deleterious for PIII processing due to the sputtering of the surface under treatment. By adjusting the power of an electron source filament made of heated tungsten, we could control the plasma potential from zero to $350 \mathrm{~V}$. In the present experiment, we worked with reduced plasma potential of the order of $100 \mathrm{~V}$.

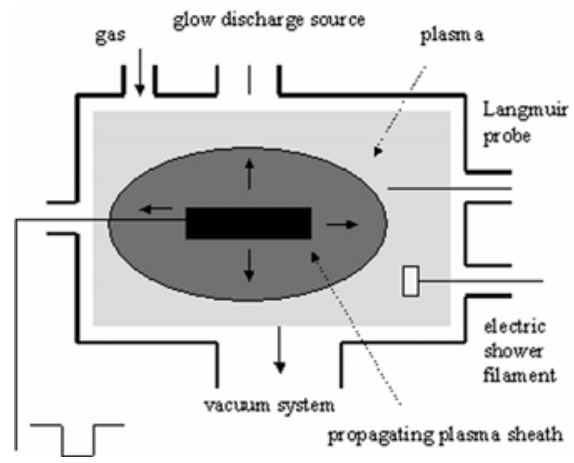

FIGURE 1. Experimental set-up for implantation tests with lower voltage and longer pulse duration.

Fig. 2 shows the experimental set-up used for the PIII treatment of UHMWPE polymer with shorter pulses and higher voltages (in the range of $1.2 \mu$ s with applied voltages of $30-50 \mathrm{kV}$ ). Because of the higher voltage applied, we use a stainless steel (SS) vacuum chamber with bigger dimensions (diameter of $58 \mathrm{~cm}$ and length of $68 \mathrm{~cm}$ ) as well as a high voltage feed- through device rated at $60 \mathrm{kV}$. To generate the high voltage pulse, a pulser of $100 \mathrm{kV} / 200 \mathrm{~A} / 100 \mathrm{~Hz}$ with fixed pulsed duration of the order of $1.2 \mu \mathrm{s}$ based on coaxial pulse forming line technology has being used, commonly known as Blumlein pulse generator [7], [8]. Similarly to the previous arrangement, we put the sample under treatment inside the chamber on an electrode support connected to the high voltage pulser and immersed in nitrogen plasma. The vacuum base pressure for the plasma production (provided by one turbo-molecular pump installed at the bottom of the vacuum chamber) was around $8.0 \times 10^{-6} \mathrm{mbar}$ with a typical working pressure of about $5.0 \times 10^{-3}$ mbar. We produced the plasma by means of a pulsed glow discharge using the same high voltage pulse applied to the target. Again, to start the pulsed glow discharge we used an electric shower filament made of thin tungsten wire of $0.2 \mathrm{~mm}$ diameter. To reduce plasma potential for avoiding surface sputtering (which could compromise the implantation process), we adjusted the AC voltage of the electric shower filament to approximately $11 \mathrm{~V}$. A pink luminescence emitted from the nitrogen glow discharge through a glass window at the chamber top indicated the plasma production.

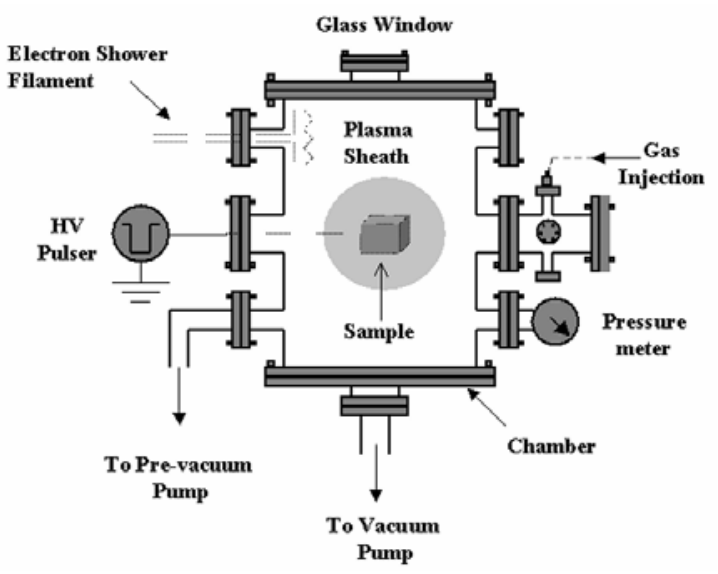

FIGURE 2. Experimental set-up for implantation tests with higher voltage and shorter pulse duration.

In both cases, we treated the polymer samples $(25$ $\mathrm{mm}$ in diameter $\times 5 \mathrm{~mm}$ in length) by putting them on a metallic sample holder to a stainless steel rod, which was linked to the pulser via a high voltage copper feed-trough device. Because of the isolation properties of the polymer, a thin wire grid with $70 \%$ transparency (connected to the high voltage potential of the holder) was laid near the sample ( $2 \mathrm{~mm}$ of distance) to attract the ions from the plasma. Since polymers are nonconducting materials and do no tolerate elevated temperature increase, thermal diffusion of the ions is 
not important and, hence, pulse repetition frequency was limited in the range of hundreds of $\mathrm{Hz}$, more specifically $100 \mathrm{~Hz}$ for both cases with higher and lower voltages.

\section{EXPERIMENTAL RESULTS}

The PIII results obtained so far with UHMWPE treatment have demonstrated that implantation process with higher energy ions can play an important role on getting implanted surfaces that are more homogenous. Experiments performed with UHMWPE, using applied lower voltages $(5 \& 15 \mathrm{kV})$ with long pulse duration (of the order of $10 \mu \mathrm{s}$ ), have shown that ion energy represents a key parameter on the dehydrogenation of polymer surface. Dehydrogenation correlates directly with cross-linked bond formation, which is related to DLC formation on polymer surface. Figs. 3 and 4 show the Raman shift for the UHMWPE treated by PIII. Each figure contains three Raman shifts for three different implanted locations in the sample. We chose the locations in order to cover the implanted sample surface, from one side to another. In this way, it was possible to realize that the DLC formation, which is correlated to the ratio of the areas under the two shoulders, one centered on $1330 \mathrm{~cm}^{-1}$ and the other on $1560 \mathrm{~cm}^{-1}$, is more homogenous when the pulse amplitude is higher. When the pulse amplitude is low, the discrepancies among the Raman shifts are visible, as we can see in the Fig 3. Theses discrepancies are a result of the shadowing effects of the grid placed in front of the polymer during the ion implantation. Since the metal grid is $2 \mathrm{~mm}$ away from the polymer surface, some of the ions extracted from the plasma with a much thicker sheath are stopped at the grid lines and others go directly through the grid apertures. Hence, at places where correspond to the shadow of the grid lines, the Raman intensity is lower because of the lower implantation doses. However, if the voltage is increased the ions tends to spread across the shadow regions due to the charging effect, leading to an implanted surface with more homogeneous properties illustrated by the DLC formation identified in Raman spectroscopy as shown in Fig. 4. Beyond the pulse amplitude, the surface homogeneity has also a strong dependence on the length of the pulse. For instance, at higher energies $(30 \mathrm{kV} \& 45 \mathrm{kV})$, but with very short pulses of about $1.2 \mu \mathrm{s}$, the same correlation, as shown in Figs. 5 and 6, can be observed for these results if we compare them to that obtained for lower energies $(5 \mathrm{kV}$ $\& 15 \mathrm{kV})$. Fig. 5 shows the Raman spectroscopy for ion energies of about $30 \mathrm{kV}$ obtained for eight location points varying along the sample surface during an exposure time of $120 \mathrm{~min}$, while Fig. 6 gives the same diagnostic for higher energies up to $45 \mathrm{kV}$ measured in fourteen different location points during an exposure time of 60 min. In Fig. 5 with lower energy, we note less homogeneity on the surface when compared to that of Fig. 6. As before, for the previous case with longer pulse, this is explained by the fact that surface charging is induced by higher voltage application. Nevertheless, the same homogeneity is obtained at lower voltages around $15 \mathrm{kV}$ with application of a pulse with longer duration (of the order of $10 \mu \mathrm{s}$ as shown in Fig. 4) instead of a higher voltage with shorter pulse duration. This means that surface homogeneity is also dependent on pulse duration since the plasma sheath formed around the target has more time to induce the spreading of the ions over the surface shadow regions, increasing the surface charging and homogeneity. Again, for Figs. 5 and 6 we observe the same DLC formation with both characteristic shoulders in the graphics around Raman wavelength shifts of $1330 \mathrm{~cm}^{-1}$ and $1560 \mathrm{~cm}^{-1}$ corresponding respectively to the $\mathrm{D}$ and $\mathrm{G}$ peaks.

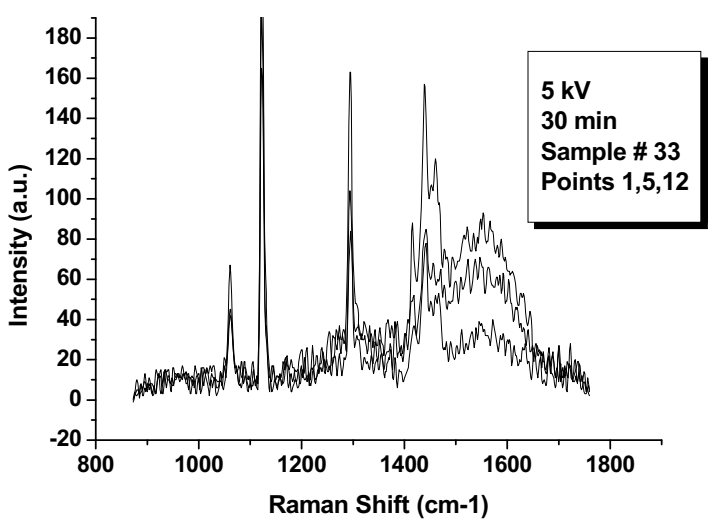

FIGURE 3. Raman shift for less energetic ions at $5 \mathrm{kV}$ with long pulse duration $(10 \mu \mathrm{s})$.

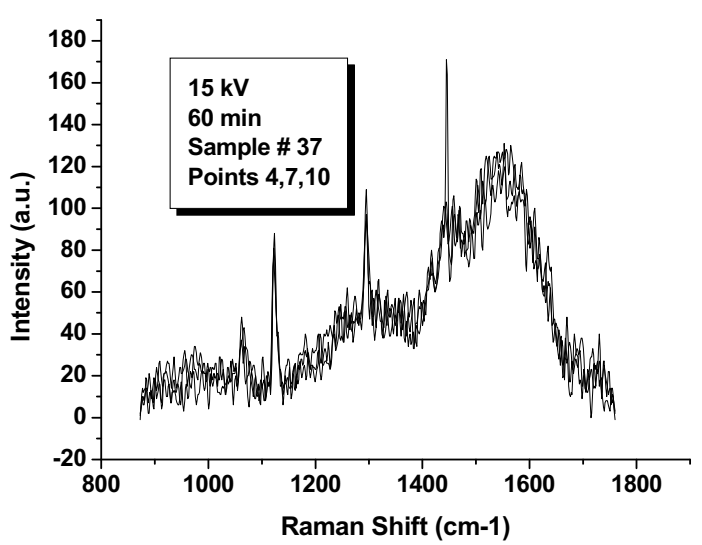

FIGURE 4. Raman shift for less energetic ions at $15 \mathrm{kV}$ with long pulse duration $(10 \mu s)$. 


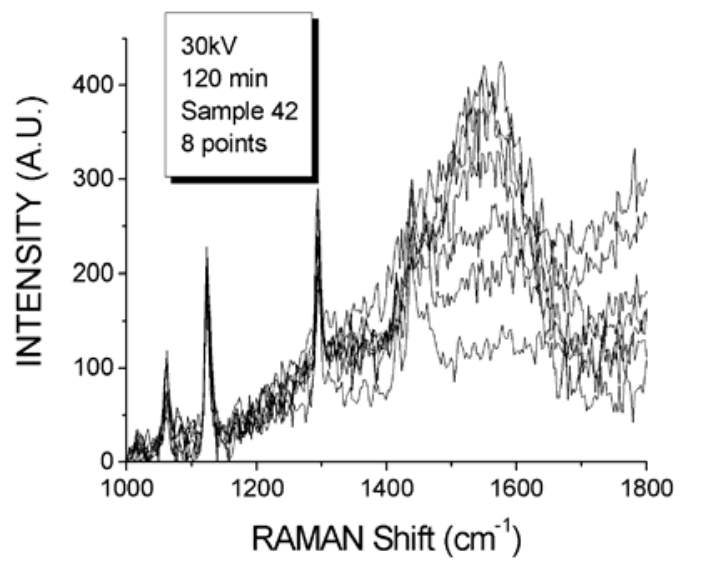

FIGURE 5. Raman shift for high-energy ions at $30 \mathrm{kV}$ with shorter pulse duration $(1.2 \mu \mathrm{s})$.

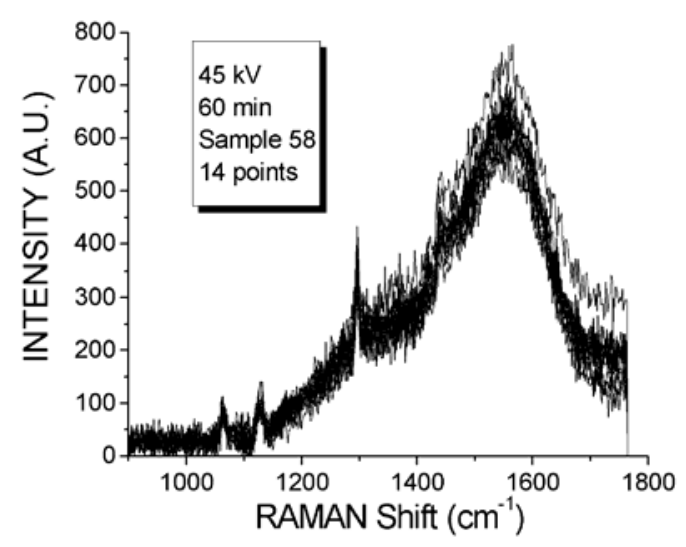

FIGURE 6. Raman shift for high-energy ions at $45 \mathrm{kV}$ with shorter pulse duration $(1.2 \mu \mathrm{s})$.

\section{CONCLUSIONS}

The results obtained so far have demonstrated that high-energy ions can be implanted on polymer surface structure without the formation of precipitates by selfclustering, and lead to cross-linked bonds among polymeric chains. Surface diagnostics by Raman spectroscopy indicate the formation of a more rigid structure on polymer surface, which could be identified as being Diamond-Like Carbon (DLC). A more homogeneous DLC formation on the polymer surface can alter the material properties, producing increased hardness factor with reduced friction coefficient, which would be ideal for applications of UHMWPE in prosthesis devices. To verify this statement, hardness and friction measurements should be performed. Moreover, the results indicate that the homogeneity and intensity of DLC formation on polymer surface is directly dependent on both duration and amplitude of the pulse provided by the high voltage pulser. In the case of pulse amplitude, this means that this dependence is directly related to the energy of the implanted ion species. Because of the need of the electrode grid structure near the polymer surface during the implantation process of nonconducting materials, non-homogeneous implantation can occur in the grid shadows over the sample surface. Obviously, this can be circumvented by applying higher voltages with short or long pulses as illustrated by the experimental results obtained. In view of that, our group has found interesting achievements when implanting UHMWPE samples with more energetic ions, especially in the range of $30-45 \mathrm{kV}$. Moreover, the HV Blumlein pulser of the second experimental set-up (see section II) has shown excellent prospects for the future studies of implantation process with high-energy ions since it can theoretically provide pulses with amplitudes up to $100 \mathrm{kV}$.

\section{ACKNOWLEDGMENTS}

The authors would like to thank Research Foundation of the Sao Paulo State (FAPESP) for its financial support. Other research funding agencies (MCT \& CNPq) are also acknowledged.

\section{REFERENCES}

1. S. Han, Y. Lee, H. Kim, G. -H. Kim, J. Lee, J. -H. Ion, and G. Kim, Surface \& Coatings Technology 93, 1997, pp. 261-264.

2. R.J. Adler, R.J. Richter-Sand, E.J. Clark, and C.W. Gregg, J. Vac. Sci. Technol. B 17(2), 1999, pp. 883-887.

3. A.R. Marcondes, M. Ueda, K.G. Kostov, A.F. Beloto, N.F. Leite, G.F. Gomes, and C.M. Lepienski, Brazilian Journal of Physics 34(4B), 2004, pp. 1667-1672.

4. I.H. Tan, M. Ueda, R.S. Dallaqua, J.O. Rossi, A.F. Beloto, M.H. Tabacnicks, N.R. Dermaquette, and Y. Inoue, Surface \& Coatings Technology 186, 2004, pp. 234-238.

5. J.O. Rossi, M. Ueda, and J.J. Barroso, Surface \& Coatings Technology 136, 2001, pp. 43-46.

6. J.O. Rossi, M. Ueda, J.J. Barroso, and V.A. Spassov, IEEE Transactions on Plasma Science 28(5), 2000, pp. 1392-1396.

7. J.O. Rossi, I.H. Tan, and M. Ueda, Nuclear Instruments and Methods in Physics Research B 242, 2006, pp. 328331.

8. J.O. Rossi, M. Ueda, and J.J. Barroso, IEEE Transactions on Plasma Science 30(5), 2002, pp. 16221626. 